\title{
NUMERICAL SIMULATION FOR ENERGY HARVESTING OF PIEZOELECTRIC FLAG IN UNIFORM FLOW
}

\author{
Song, R. J. ; Hou, C. W. ; Shi, Z. C. ${ }^{* * * * * *}$; Yang, X. H. ; Jiang, S. B. .*,\# \& Jia, J. D. ${ }^{* * * *}$ \\ *School of Mechanical Engineering, Shandong University of Technology, Zibo 255000, China \\ ${ }^{* *}$ College of Mechanical and Electronic Engineering, Shandong University of Science and \\ Technology, Qingdao 266590, China \\ ${ }^{* * *}$ School of Chemical Engineering and Energy, Zhengzhou University, Zhengzhou 450004, China \\ ***** School of Civil and Environmental Engineering, Nanyang Technological University, 639798, \\ Singapore \\ E-Mall: jiangshoubo@ sdust.edu.cn (\# Corresponding author)
}

\begin{abstract}
Piezoelectric energy harvesters (PEHs) have been investigated for decade years for powering low energy consumed electric devices. However, research of PEH more focuses on the structure and energy capture performance, ignoring the interaction between the PEHs and environmental vibration. In order to investigate energy harvesting performance of PEHs in uniform flow, this study presented a two-dimensional (2D) simulation method. The fluid kinematics was simulated by the discrete lattice Boltzmann equation. Fluid effecting on the piezoelectric flag were handled by immersed boundary method (IBM). Coupled with Euler-Bernoulli beam and piezoelectric theory, the full-coupled fluidstructure-electric (FSE) was established by using the immersed boundary-lattice Boltzmann method. Results indicate that due to the simple boundary treatment and time saving calculation of the lattice Boltzmann method (LBM) and IBM, the numerical method is superior efficiency for the FSE problems. The identified peak energy conversion efficiencies for various velocities are significant to explore the optimum structure of piezoelectric flags in various fluid situations.

(Received in February 2019, accepted in May 2019. This paper was with the authors 1 month for 1 revision.)
\end{abstract}

Key Words: Numerical Simulation, Piezoelectric Flag, Energy Harvesting, Flow

\section{INTRODUCTION}

The phenomena of flow-induced vibration are widely distributed in nature, such as the flapping flags and leaves in the wind, the flapping waterweeds in the flowing water [1]. The flow kinetic energy can be converted into electricity to power electronic devices. Meanwhile, piezoelectric [2], electrostatic [3], electret [4] and electromagnetic [5, 6] are the main transduction mechanisms. And the piezoelectric energy harvesting is the most popular one among them. Piezoelectric energy harvester (PEH) can be stimulated by the flow-induced vibration to translate the flow kinetic energy into electricity. All above energy harvesters were investigated under based-excitation. However, the vibration condition of the ambient environment is usually different from the based-excitation. In order to actually explore the energy harvesting performance of the harvester, coupling effects from the environment on a harvester should be considered.

As for the fluid dynamic environment, the effects from the fluid should be taken into account. Hence, for the fluid-induced piezoelectric energy harvesting (FIPEH), researchers focused on exploring the fluidic energy harvester with respect to various fluid conditions. However, the results may be worked out with great error via different numerical methods. Therefore, the dynamic response and energy harvesting performance of piezoelectric flags in uniform flow can be numerically analysed efficiently through some kinds of methods, which must be studied to simulate the results more accurately. 


\section{STATE OF THE ART}

In recent years, many researchers have focused on the piezoelectric energy harvesting from flow-induced vibration [7-9], for instance, wake-induced vibration (WIV) and flutter. For the WIV, a practical eel-shaped PEH was first developed to convert water kinetic energy into electricity by Taylor et al. [10]. From then on, some works of energy harvesting using piezoelectric flags have been investigated with unsteady turbulent vortex shedding from upstream bluff bodies [11-13]. Wang et al. [13] investigated the different section of bluff bodies on energy harvesting efficiency. In addition, flutter is one of most common phenomena of flow-induced vibration. Energy harvesting using flutter PEH was investigated by some researchers in uniform flow. For example, Dunnmon et al. [14] experimentally studied the nonlinear limit-cycle oscillations of the PEH to enhance power generation from aerodynamic flows. Singh et al. [15] numerically analysed the energy harvesting performance of piezoelectric flag under the effect of fluttering modes. What's more, multi-stable and nonlinear energy harvesters are also both of hot research of enhancing energy harvesting performance. For instance, Huang et al. [16] and Zhou et al. [17, 18] studied the multi-stable energy harvesters and their nonlinear dynamic energy harvesting performance. And there are also some scholars studied on PEH $[19,20]$ as well as used inverse piezoelectric effect for motor driving [21, 22].

Akcabay and Young [23] presented a numerical method to investigate the energy harvesting performance of a single piezoelectric flag. It's worth noting that the responses of fluid-structure-interaction (FSI) in all above studies are solved using the Navier-Stokes (N-S) equation directly, which are meaningful. However, the numerical method is tedious and timeconsuming for calculating the result of energy harvesting performance. An alternative and robust method for fluid-flow simulation is the lattice Boltzmann method (LBM), which is an approach based on the particle kinetics. In the LBM, a popular scheme for FSI is based on the "bounce-back" rule to enforce the no-slip and no-penetration conditions at the solid surface [24].

The remainder of this study is organized as follows. Section 3 introduces the physic mode and numerical method. A novel two-dimensional (2D) coupling numerical method is proposed for simulating energy harvesting performance of piezoelectric flag in uniform flow. Section 4 analyses the result with different parameter setting. The dynamic response and energy harvesting performance of piezoelectric flags in uniform flow are discussed in detail. Section 5 summarizes the conclusions.

\section{METHODOLOGY}

\subsection{Problem statement}

In order to uncover the essence of physic mode and to simplify the numerical computation, the mathematical model is derived by the following assumption. Fluid can be described in two-dimensional (2D) with the characters of laminar, viscous, Newtonian and incompressible. According to above assumptions, Lattice Boltzmann equation can be introduced to describe the fluid instead of directly solving the N-S equation [25, 26], penalty immersed boundary method can be introduced to solve the FSI response [24, 27], and Euler-Bernoulli beam theory coupled with piezoelectric equation can be used to calculate the deformations and energy harvesting performance of piezoelectric flag $[2,28]$.

In this numerical method, a discrete lattice Boltzmann equation of a single relaxation time model with two-dimensional (2D), nine-speed (D2Q9) is used to govern the fluid domain, which can be presented in references $[25,29,30]$. 


$$
f_{i}\left(x+e_{i} \Delta t, t+\Delta t\right)-f_{i}(x, t)=-\frac{1}{\tau}\left[f_{i}(x, t)-f_{i}^{e q}(x, t)\right]+\Delta t G_{i}
$$

where, $f(x, t)$ represents the distribution function for particles with velocity $e_{i}$ at the position $x$ and time $t, f_{i}^{e q}(x, t)$ represents the equilibrium distribution function, $\tau$ is the dimensionless relaxation time, $\Delta t$ is the size of the time step, and $G_{i}$ represents the body force effect on distribution function, and the details above can be found in references [25, 29, 30].

Fig. 1 shows the three-dimensional model of the piezoelectric flag in the flow field. Fig. 2 shows the schematic of piezoelectric flag and its equivalent circuit.

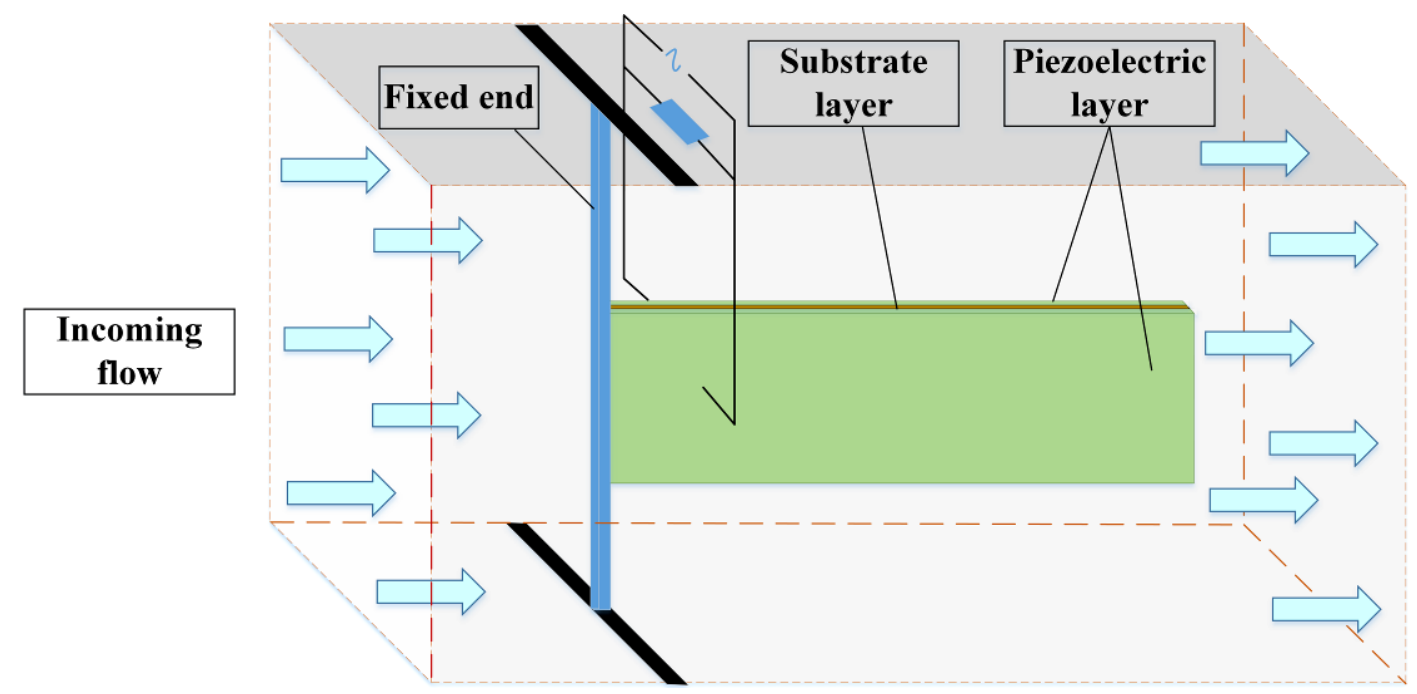

Figure 1: Three-dimensional model of the piezoelectric flag.

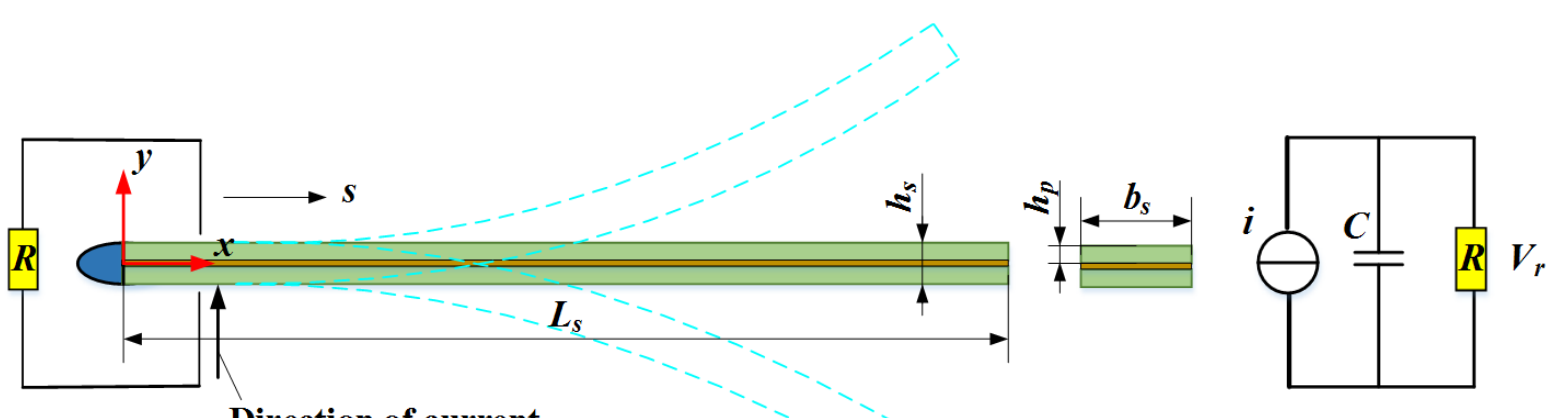

Direction of current

Figure 2: Schematic of the flexible piezoelectric flag (left) and the equivalent circuit of piezoelectric flag (right).

As shown in Fig. 2 (left), the piezoelectric flag consists of two outer piezoelectric layers adhered to an inner substrate layer and is fixed in the upstream in uniform flow. The thickness of piezoelectric layer and total flag are $h_{p}$ and $h_{s}$, respectively. $H_{s}$ is assumed to be equal to $2 h_{p}$ by ignoring the thickness of the substrate layer. The piezoelectric flag is assumed to be inextensibility in s-direction and $s$ is the Lagrangian coordinate along the length of flag. $K_{b}, L_{s}$ and $b_{s}$ are the bending stiffness, length and width, respectively. $i, V_{r}$, and $C$ are individually transient current, transient voltage, and equivalent capacitance of the piezoelectric flag. $R$ is the external resistance, as shown in Fig. 2 (right).

On the basis of physical model, the partial differential governing equation with the fluid forced has been expressed in as follows. 


$$
m_{s} \frac{\partial^{2} X_{s}}{\partial t^{2}}=T_{s} \frac{\partial^{2} X_{s}}{\partial s^{2}}-K_{b} \frac{\partial^{4} X_{s}}{\partial s^{4}}+\Theta_{s} V_{r} \frac{d}{d s}\left[\delta(s)-\delta\left(s-L_{s}\right)\right]-F_{f}
$$

where, $\delta$ is a Dirac-Delta function, $m_{s}$ is the mass per unit length of the flag, equal to $p_{s} h_{s}, X_{s}$ is the position vector, $T_{s}$ is the tension force determined by the constraint inextensibility, $K_{b}$ is the bending rigidity, and equal to $E h_{s}^{3} / 12, \Theta_{s}$ is the piezoelectric coupling term and equal to $e_{31} h_{s} / 4, V_{r}$ is output voltage[31, 32].

The governing equivalent circuit equation of the flag can be written as [2].

$$
C \frac{\partial V_{r}}{\partial t}+\frac{V_{r}}{R}+\Theta_{s} b_{s} \int_{0}^{L} \frac{\partial^{3} X_{s}}{\partial s^{2} \partial t} d s=0
$$

where, $C=\varepsilon L_{s} b_{s} / h_{s} . T$ is the flapping cycle. $\rho_{s}$ is the flag density. $\rho$ is the piezoelectric electric permittivity.

In order to easily calculate, Eqs. (2) and (3) can be non-dimensionalized by introducing the following characteristic scales: $L_{s}$ for length, $U_{f}$ for velocity, $L_{s} / U_{f}$ for time $t$ and time cycle $T, \rho_{f} U_{f}^{2}$ for force $F_{f}, \rho_{f} U_{f}^{2} L_{s}$ for the tension force $T_{s}$. Hence, the governed equations of the whole energy harvesting system can be expressed as follows.

$$
\begin{gathered}
S \frac{\partial^{2} X}{\partial t^{2}}=T \frac{\partial^{2} X}{\partial s^{2}}-K \frac{\partial^{4} X}{\partial s^{4}}+\Theta V \frac{d}{d s}[\delta(s)-\delta(s-1)]-F \\
\frac{1}{h} \frac{\partial V}{\partial t}+\frac{V}{R^{\prime}}+\Theta^{\prime} \int_{0}^{1} \frac{\partial^{3} X}{\partial s^{2} \partial t} d s=0
\end{gathered}
$$

where, $S=m_{s} /\left(\rho_{f} L_{s}\right)$ is the ratio of the solid inertial force to fluid inertial force, $K=K_{b} / \rho_{f} L_{s}^{3} U_{f}^{2}$ is the ratio of the structural bending stiffness force to fluid inertial force. The dimensionless thickness $h$ is scaled by $L_{s}$; the dimensionless voltage $V$ is scaled by $L_{s} U_{f}\left(\rho_{f} / \varepsilon\right)^{1 / 2}$; the piezoelectric coupling terms $\Theta$ is scaled by $L_{s} U_{f}\left(\rho_{f} \varepsilon\right)^{1 / 2} ; \Theta^{\prime}=\Theta b_{s} ;$ the dimensionless resistivity $R^{\prime}$ is scaled by $L_{s} /\left(\varepsilon U_{f} b_{s}\right)$, the dimensionless power $P$ is scaled by $\rho_{f} U_{f}^{3} L_{s} b_{s} \varepsilon^{1 / 2}$. Besides, the Reynolds number $R e$ is defined by $R e=\rho_{f} L_{s} U_{f} / \mu_{f}$.

The clamped-free boundary conditions can be expressed as follows.

$$
\begin{aligned}
& s=0, \frac{\partial X}{\partial s}=0 \\
& s=1, K \frac{\partial^{2} X}{\partial s^{2}}-\Theta V[H(s)-H(s-1)]=K \frac{\partial^{3} X}{\partial s^{3}}=\Theta V[\delta(s)-\delta(s-1)]=T=0
\end{aligned}
$$

The IBM is extended here to solve the FSI. The Lagrangian force $F$ exerted in Eq. (4) can be generated by references $[24,33]$.

$$
F=\alpha\left(X-X_{i b}\right)+\beta\left(U-U_{i b}\right)
$$

where $\alpha$ and $\beta$ are positive constants. $U=d X / d t$ is the velocity of the flag. $U_{i b}$ is the IB velocity expressed as follows.

$$
U_{i b}(s, t)=\int U(s, t) \delta(x-X(s, t)) d x
$$

where, $u$ is the velocity of fluid. $X_{i b}$ is the position integrated by using $U_{i b}$.

The Lagrangian force spreads to the nearby Eulerian grid and the body force $B$ can be expressed as follows.

$$
B=\int F(s, t) \delta(x-X(s, t)) d s
$$


Lagrangian force $F$ in Eq. (4) can be calculated by Eq. (7). The output voltage $V$ and power $P$ can be obtained by Eqs. (4) and (5). Body force $B$ in Eq. (1) can be gained by Eq. (9) using Eq. (7). Therefore, the fully coupled of FSE is achieved.

\subsection{Numerical process}

An unformed Lagrangian grid is used in this study. The indices start from the fixed end $(i=0)$ to the free end $(i=N) . D_{s}, D_{s s}, D_{s s s}$, are the first, second and third order derivatives of the arc length $s$ respectively. For the arbitrary variable $\varphi$, where, $D_{s}^{0} \phi, D_{s}^{-} \phi, D_{s}^{+} \phi, D_{s}^{+} D_{s}^{-} \phi$ denote the centre, downwind, upwind, second-order difference approximations, which can be expressed as follows.

$$
\begin{aligned}
& D_{s}^{0} \phi=(\phi(s+\Delta s / 2)-\phi(s-\Delta s / 2)) / \Delta s \\
& D_{s}^{-} \phi=(\phi(s)-\phi(s-\Delta s)) / \Delta s \\
& D_{s}^{+}=(\phi(s+\Delta s)-\phi(s)) / \Delta s \\
& D_{s}^{+} D_{s}^{-} \phi=(\phi(s+\Delta s)-2 \phi(s)+\phi(s-\Delta s)) / \Delta s^{2}
\end{aligned}
$$

The time $t$ can be applied with the same rules.

\subsection{Discretization of governing equation}

The solid governing equation consists of tension force $F_{t}$, bend force $F_{b}$, electromechanical coupling force $F_{V}$ and inertial force $F_{a}$.

The tension force $F_{t}=T \partial^{2} X / \partial s^{2}$ of Eq. (4) can be discretized as follows.

$$
\left(F_{t}\right)_{i}=\left\{\begin{array}{l}
{\left[D_{s}\left(T D_{s} X\right)\right]_{i}, i=1,2, \ldots, N-1} \\
\frac{\left(T D_{s} X\right)_{i}-\left(T D_{s} X\right)_{i-1 / 2}}{0.5 \Delta s}, i=N
\end{array}\right.
$$

The bend force $F_{b}=-\partial^{2}\left(K \partial^{2} X / \partial s^{2}\right) / \partial s^{2}$ can be discretized as follows.

$$
\left(F_{b}\right)_{i}=\left\{\begin{array}{l}
-\left[D_{s}^{+} D_{s}^{-}\left(K D_{s s} X\right)\right]_{i}, i=1,2, \ldots, N-1 \\
-K\left[D_{s}^{+}\left(D_{s s s} X\right)\right]_{i}, i=N
\end{array}\right.
$$

The electromechanical coupling force $F_{V}=\Theta V d[\delta(s)-\delta(s-1)] / d s$ can be discretized.

$$
\left(F_{V}\right)_{i}^{n}=\Theta V^{n} \frac{\left[\delta_{h 1}(i \Delta s)-\delta_{h 1}(i \Delta s-1)\right]}{\Delta s}, i=0,1,2, \ldots, N
$$

The inertial force $F_{a}=S \partial^{2} X / \partial t^{2}$ can be discretized as follows.

$$
\left(F_{a}\right)_{i}^{n}=\left(S D_{t}^{+} D_{t}^{-} X_{i}\right)_{i}^{n}, i=0,1,2, \ldots, N
$$

At fixed end $(i=0),\left(F_{a}\right)_{0}^{n}=0$, according to the above discretized terms, Eq. (4) can be summarized as follows.

$$
\left(F_{a}\right)_{i}^{n}=\left(F_{t}\right)_{i}+\left(F_{b}\right)+\left(F_{V}\right)_{i}^{n}-F_{i}^{n}, i=0,1,2, \ldots, N
$$

\subsection{Discretization of electric governing equation}

The electric governing equation of Eq. (5) can be summarized as follows.

$$
\frac{1}{h} \frac{V^{n+1}-V^{n}}{\Delta t}+\frac{V^{n}}{R^{\prime}}+\Theta^{\prime} \sum_{i=0}^{N} \frac{\left(D_{s s} X\right)_{i}^{n+1}-\left(D_{s s} X\right)_{i}^{n}}{\Delta t}=0, i=0,1,2, \ldots, N
$$


From Eq. (16), the voltage at $(n+1)^{\text {th }}$ time $V^{n+1}$ can be obtained as follows.

$$
V^{n+1}=\frac{R^{\prime}}{R^{\prime}+h \Delta t} V^{n}+\frac{R^{\prime} h \Delta t(I) i}{R^{\prime}+h \Delta t}, i=0,1,2, \ldots, N
$$

Besides, in the $t=0$, the voltage $V_{1}=0$.

\subsection{Discretization of interaction equation}

Eq. (7) can be discretized as follows.

$$
\begin{aligned}
& F_{i}^{n}=\alpha\left(X_{i}^{n}-\sum_{j=1}^{n}\left(U_{i b}\right)_{i}^{j} \Delta t\right)+\beta\left(U_{i}^{n}-\left(U_{i b}\right)_{i}^{n}\right) \\
& U_{i}^{j}=\frac{\left(X_{i}^{j}-X_{i}^{j-1}\right)}{\Delta t} \\
& \left(U_{i b}\right)_{i}^{j}=\sum_{x_{m l} \in g_{h}} u_{m l}^{j} \delta_{h}\left(x_{m l}-X_{i}^{j}\right) h^{2}, \quad i=0,1,2, \ldots, N, j=1,2, \ldots, n
\end{aligned}
$$

where, $h=\Delta x=\Delta y$ is the grid size of the fluid mesh, $g_{h}$ is the computational domain.

Eq. (9) can be discretized as follows.

$$
B=\sum_{1}^{i=N} F_{i}^{n} \delta_{h}\left(x_{m l}-X_{i}^{n}\right) \Delta s, x_{m l} \in g_{h}
$$

\subsection{Summary of coupling progress}

The general coupling progress can be summarized as follows.

(1) At time $t$, there are parameters of fluid velocity $U^{n}$, pressure $P^{n}$, density $\rho$, the position of the piezoelectric flag $X^{n}$ and transient voltage $V^{n}$. Interpolating the $U^{n}$, use the IBM to obtain the $U_{i b}$ and the Lagrangian force $F$ by Eqs. (7) and (8).

(2) Spreading the Lagrangian force $F$ to the nearby Eulerian grid by Eq. (9), and body force $B$ is obtained. New information of the fluid is updated.

(3) Substituting the Lagrangian force $F$ and transient voltage $V^{n}$ into Eqs. (4) and (5) to obtain the piezoelectric flag position $X^{n+1}$ and transient voltage $V^{n+1}$. Furthermore, the output power $P^{n}$ is obtained.

(4) One step is over. The fluid velocity $U^{n+1}$, pressure $P^{n+1}$, new position of flag $X^{n+1}$ and transient voltage $V^{n+1}$ can be obtained from steps (2) and (3) and all above data will be used in the next step $t+\Delta t$.

\section{RESULT ANALYSIS AND DISCUSSION}

The dynamic response and energy harvesting performance of piezoelectric flags in uniform flow can be achieved by means of the presented methods. As an application, a piezoelectric flag was investigated numerically. A flexible piezoelectric material of Polyvinylidene Fluoride (PVDF) was used in this work. Hence, calculation parameters are listed: elasticity modulus $E$ of $3 \mathrm{GP}$, the density $\rho_{s}$ of $1700 \mathrm{~kg} / \mathrm{m}^{3}$, piezoelectric electric permittivity $e_{31}$ of $-0.06 \mathrm{c} / \mathrm{m}^{2}$, air density $\rho_{f}$ of $1.209 \mathrm{~kg} / \mathrm{m}^{3}$, length $L_{s}$ of $100 \mathrm{~mm}$ and width $b_{s}$ of $10 \mathrm{~mm}$ and so on. Furthermore $h=h_{s} / L_{s}$ is the specific analysis parameter, and the flow velocities of $U_{1}=3 \mathrm{~m} / \mathrm{s}, U_{2}=4 \mathrm{~m} / \mathrm{s}, U_{3}=5 \mathrm{~m} / \mathrm{s}$ are respectively chosen as the certain velocities for investigating dynamic response of flag. It is noteworthy that, in order to compute simply, Reynolds number is artificially kept on order of $10^{2}$ level, which is about 100 times lower than the real condition, but the numerical simulation results could be agreement with the results of real condition, which have been verified [34]. 
Fig. 3 displays the vibration response of a piezoelectric flag with the parameters of velocity of $3 \mathrm{~m} / \mathrm{s}$, resistance of $3 \mathrm{M} \Omega$ and $h=5 \times 10^{-4}$. Figs. $3 \mathrm{a}$ and b show the vorticity field. In Fig. $3 \mathrm{c}$, it can be found that the flapping of flag comes into limit-cycle after a few cycles adjustment. Output alternating voltage cyclically changes with cycle $T$, as shown in Fig. $3 \mathrm{~d}$.

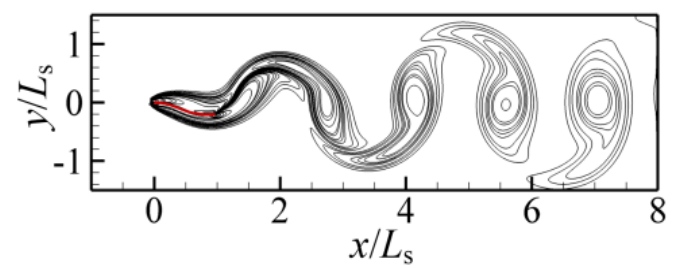

a) Vorticity field for $t U_{f} / L_{s}=38.5$

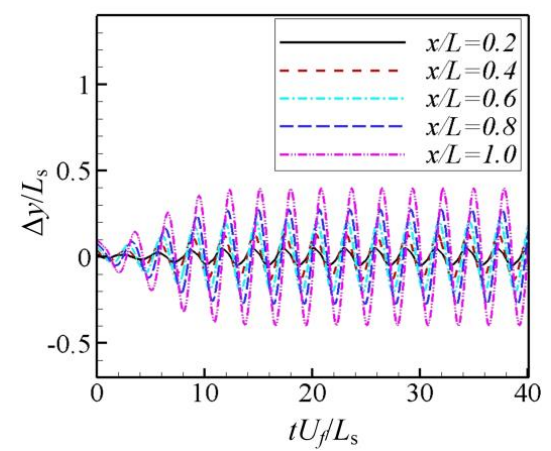

c) Displacement

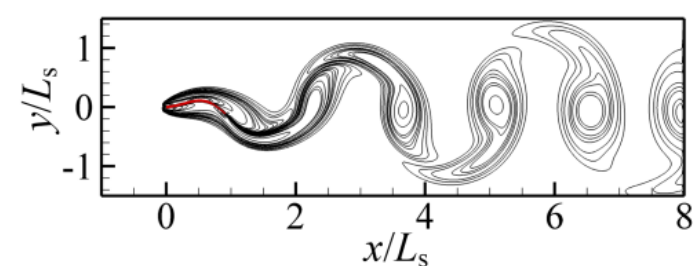

b) Vorticity field for $t U_{f} / L_{s}=39.5$

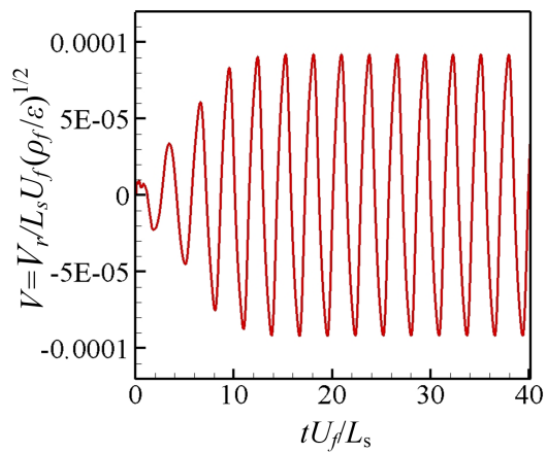

d) Output power

Figure 3: Dynamic response and voltage obtained of a piezoelectric flag.

Fig. 4 shows vibration modes of $h=1 \times 10^{-4}, h=5 \times 10^{-4}, h=10 \times 10^{-4}, h=11.5 \times 10^{-4}$, at velocity of $3 \mathrm{~m} / \mathrm{s}$. It can be found that the vibration is occurred at the free end of flag with smaller $h$. The vibration mode moves to the fixed end and the meanwhile, vibration response increases with the increase of $h$. While, the vibration response decreases when the $h$ keeps growing, which indicates maximum vibration response and peak output power can be achieved with resonance vibration.
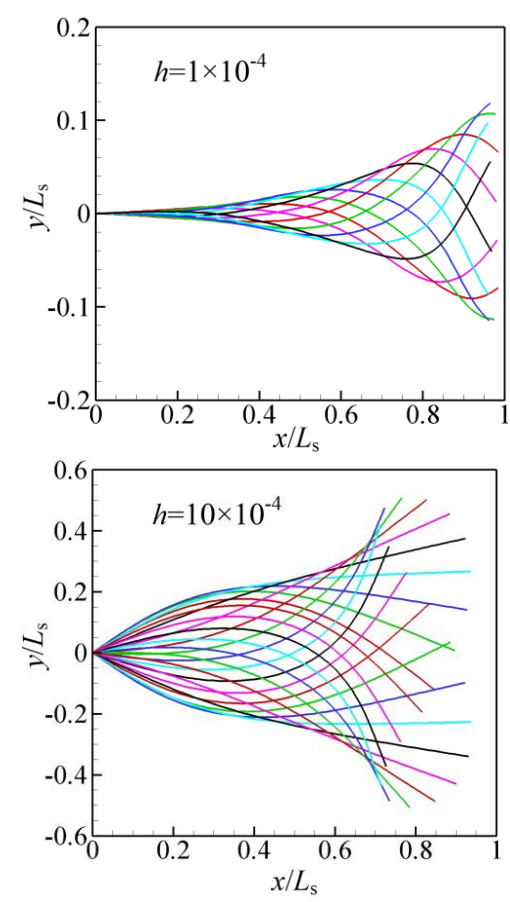
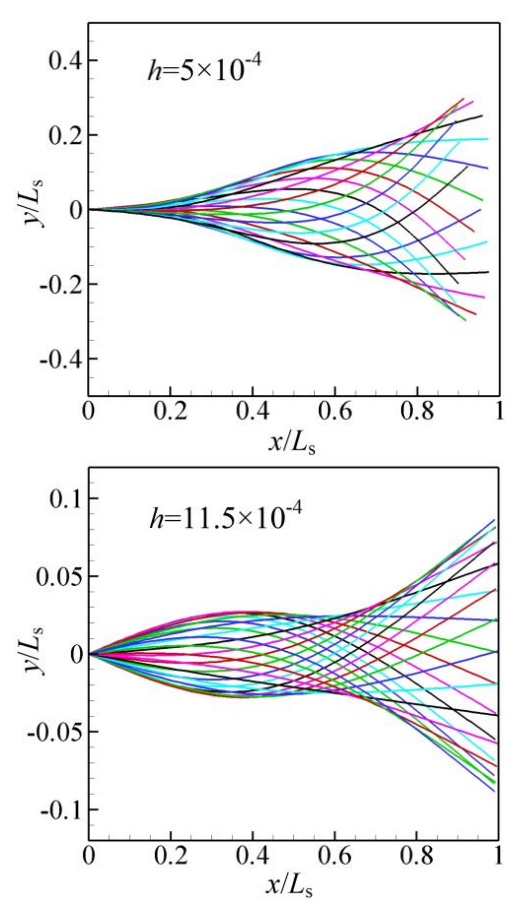

Figure 4: Vibration mode of various $h$. 
Dimensionless frequency $f$ is scaled by $U_{f} / f_{0} L_{s}$, non-dimensional amplitude $A$ is scaled by $L_{s} / A_{s}$ for the sake of the dynamic characteristic piezoelectric flag, where $f_{0}$ is real frequency and $A_{s}$ is real amplitude of piezoelectric flag. Fig. 5 illustrates the vibration frequency and amplitude at various $h$. It can be found that the frequency $f$ is high and amplitude $A$ is small in the small $h$. The frequency $f$ decreases and amplitude $A$ increases with the increase of $h$. When $h$ respectively increases to $9 \times 10^{-4}$ for $U_{1}, 11 \times 10^{-4}$ for $U_{2}, 13 \times 10^{-4}$ for $U_{3}$, the sympathetic vibration occurs with maximum amplitude $A$ under flow stimulation. In the larger $h$, the flowing fluid is unable to excite and maintain the piezoelectric flag to vibration, which the amplitude $A$ appears to down to zero.

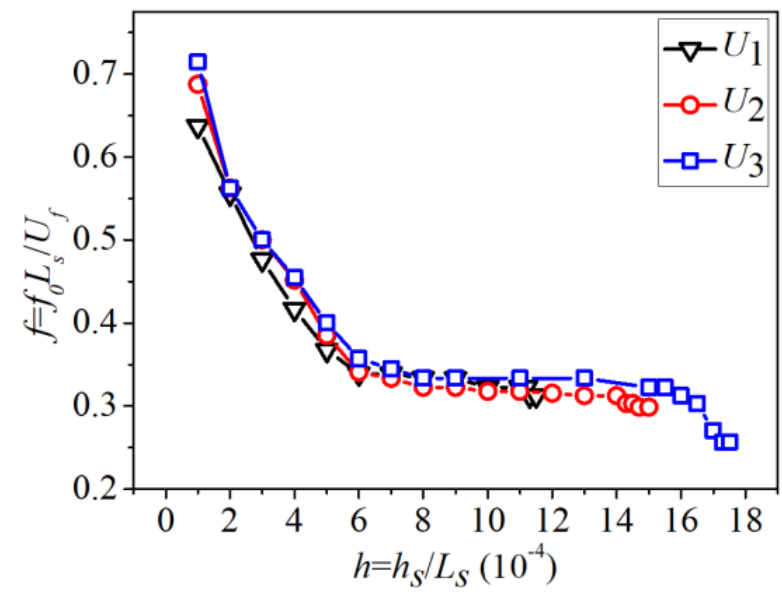

a) Vibration frequency

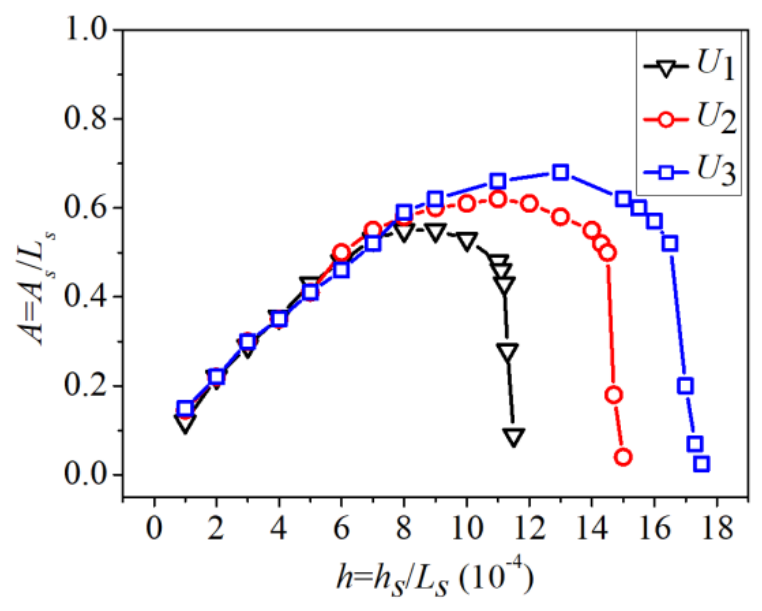

b) Vibration amplitude

Figure 5: Vibration frequency and amplitude as the function of $h, U_{1}=3 \mathrm{~m} / \mathrm{s}, U_{2}=4 \mathrm{~m} / \mathrm{s}, U_{3}=5 \mathrm{~m} / \mathrm{s}$.

There are three parameters in the energy harvesting system, including output voltage $V$, output power $P$ and energy conversion efficiency $\eta$. The $\eta$ can be expressed as $P / P_{f}$ and $P_{f}$ is the non-dimensional fluid kinetic energy, which can be obtained by $P_{f}=P_{f r} /\left(\rho_{f} U_{f}^{3} L_{s} b_{s} \varepsilon^{1 / 2}\right)$, and $P_{f r}=A_{s} L_{s} b_{s} \rho_{f} U_{f}^{3}$.

Here, the energy conversion efficiency $\eta$ can be used to evaluate the energy harvesting performance of piezoelectric flag. According to the equivalent circuit in Fig. 2 b, maximum power can be theoretically obtained when external resistance $R$ is equal to the internal impedance of piezoelectric flag $X_{c}$. Hence, optimal resistance $R=X_{c}=1 /\left(2 \pi f_{0} C_{s}\right)$ is maximum output power.

Fig. 6 a illustrates the output powers $P$ at different $h$ with optimal resistance $R$. The variation tendency of output power is same with its vibration amplitude in Fig. 5 b, where $P$ increases to maximum value and then decreases to zero in the wake of $h$ for $U_{1}, U_{2}$ and $U_{3}$, respectively. The results show that peak powers for each velocity are $P_{U 1}<P_{U 2}<P_{U 3}$ and the corresponding $h$ for peak power is $h_{U 1}<h_{U 2}<h_{U 3}$, which means piezoelectric flag with higher $h$ can generate more power by stimulating of higher velocity.

The energy conversion efficient $\eta$ can be obtained, as shown in Fig. 6 b. It can be found that there is also a peak conversion efficient for each velocity $U_{1}, U_{2}$ and $U_{3}$, respectively. Piezoelectric flag with smaller $h$ is suitable for lower-velocity flow and piezoelectric flag with higher $h$ is suited to harvest energy in higher-velocity flow. The simulation results show that optimum structure of piezoelectric flags can be explored to achieve maximum output power in various fluid velocities. 


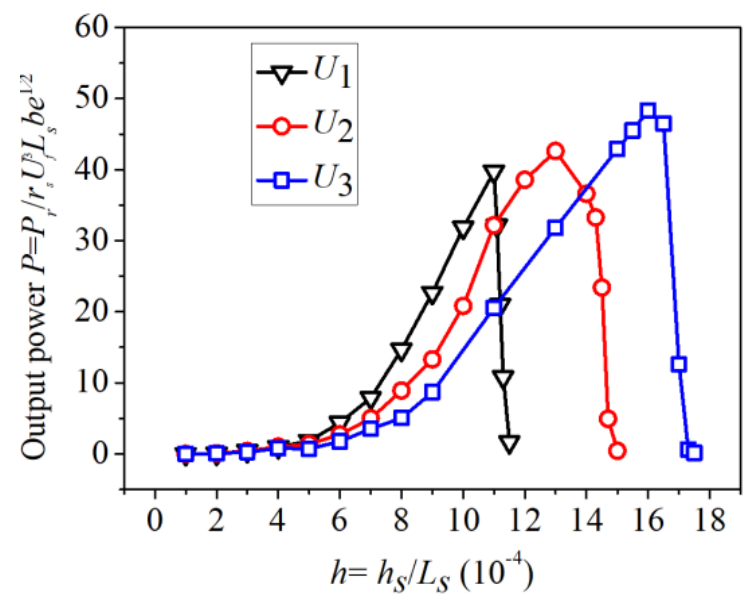

a) Power with optimal resistance

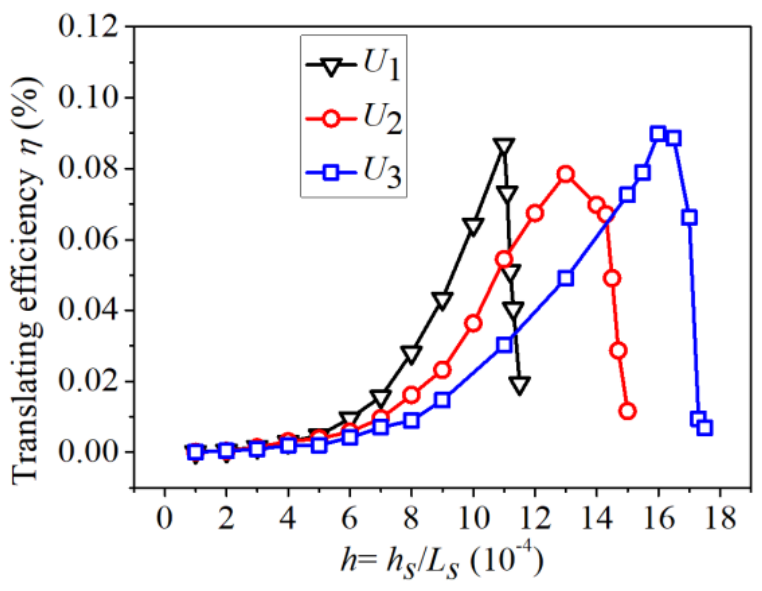

b) Conversion efficient $\eta$

Figure 6: Simulation results as a function of $h$.

\section{CONCLUSION}

In this study, a 2D simulation coupling numerical method for energy harvesting simulation using piezoelectric flag in a uniform flow is proposed. Immersed boundary-lattice Boltzmann method was introduced to solve the hydromechanics and fluid-structure interaction instead of directly solving the Navier-Stokes equation. Euler-Bernoulli beam coupled with piezoelectric theory were developed to solve the mechanical and piezoelectric effect, by which the fully coupled fluid-structure-electric interaction was achieved. Conclusions are drawn as follows.

(1) The numerical method can be used to investigate the dynamic response and energy harvesting performance of piezoelectric flag in uniform flow, which is simple, effective and time-saving.

(2) The maximum power output can be obtained by using the optimal resistances for different velocities. The frequency $f$ decreases and amplitude $A$ increases with the increase of $h$. Piezoelectric flag with higher $h$ can generate more power by stimulating of higher velocity.

(3) Piezoelectric flag with small $h$ and great $h$ are suitable for low-velocity flow and highvelocity flow, respectively, which indicates that optimum structure of piezoelectric flags, should be explored to achieve maximum output power in various fluid velocities.

The novel two-dimensional (2D) coupling numerical method is proposed for simulating energy harvesting performance of piezoelectric flag in uniform flow. And studying the energy harvesting performance of piezoelectric flag from the respect of simulating is an essential method. The study provides the advantages of simple boundary treatment and time saving calculation, which is useful for studying energy harvesting performance. However, in order to establish mathematical model, we make some assumptions, which will cause a little bit of errors. Therefore, we should take into account these errors for obtaining the accurate results.

\section{ACKNOWLEDGEMENT}

This work was supported by the National Natural Science Foundation of China (Grant No. 51705296); Shandong Provincial Natural Science Foundation, China (Grant Nos. ZR2017BEE039, ZR2017BEE025 and ZR2019BEE066); China Postdoctoral Science Foundation (Grant No. 2018M632694).

\section{REFERENCES}

[1] Tian, F.; Lu, X.; Luo, H. (2012). Onset of instability of a flag in uniform flow, Theoretical and Applied Mechanics Letters, Vol. 2, No. 2, Paper 022005, 5 pages, doi:10.1063/2.1202205 
[2] Erturk, A.; Inman, D. J. (2009). An experimentally validated bimorph cantilever model for piezoelectric energy harvesting from base excitations, Smart Materials and Structures, Vol. 18, No. 2, Paper 025009, doi:10.1088/0964-1726/18/2/025009

[3] Lallart, M.; Richard, C.; Garbuio, L.; Petit, L.; Guyomar, D. (2011). High efficiency, wide load bandwidth piezoelectric energy scavenging by a hybrid nonlinear approach, Sensors and Actuators A: Physical, Vol. 165, No. 2, 294-302, doi:10.1016/j.sna.2010.09.022

[4] Tao, K.; Tang, L.; Wu, J.; Lye, S. W.; Chang, H.; Miao, J. (2018). Investigation of multimodal electret-based MEMS energy harvester with impact-induced nonlinearity, Journal of Microelectromechanical Systems, Vol. 27, No. 2, 276-288, doi:10.1109/jmems.2018.2792686

[5] Shan, X.; Xu, Z.; Xie, T. (2013). New electromechanical coupling model and optimization of an electromagnetic energy harvester, Ferroelectrics, Vol. 450, No. 1, 66-73, doi:10.1080/ $\underline{00150193.2013 .838491}$

[6] Shan, X.; Xu, Z.; Song, R.; Xie, T. (2013). A new mathematical model for a piezoelectricelectromagnetic hybrid energy harvester, Ferroelectrics, Vol. 450, No. 1, 57-65, doi: $10.1080 / 00150193.2013 .838490$

[7] Tang, L.; Zhao, L.; Yang, Y.; Lefeuvre, E. (2015). Equivalent circuit representation and analysis of galloping-based wind energy harvesting, IEEE/ASME Transactions on Mechatronics, Vol. 20, No. 2, 834-844, doi:10.1109/tmech.2014.2308182

[8] Shan, X.; Deng, J.; Song, R.; Xie, T. (2017). A piezoelectric energy harvester with bendingtorsion vibration in low-speed water, Applied Sciences, Vol. 7, No. 2, Paper 116, 16 pages, doi:10.3390/app7020116

[9] Shan, X.; Li, H.; Yang, Y.; Feng, J.; Wang, Y.; Xie, T. (2019). Enhancing the performance of an underwater piezoelectric energy harvester based on flow-induced vibration, Energy, Vol. 172, 134-140, doi:10.1016/j.energy.2019.01.120

[10] Taylor, G. W.; Burns, J. R.; Kammann, S. A.; Powers, W. B.; Welsh, T. R. (2001). The Energy Harvesting Eel: a small subsurface ocean/river power generator, IEEE Journal of Oceanic Engineering, Vol. 26, No. 4, 539-547, doi:10.1109/48.972090

[11] Akaydin, H. D.; Elvin, N.; Andreopoulos, Y. (2012). The performance of a self-excited fluidic energy harvester, Smart Materials and Structures, Vol. 21, No. 2, Paper 025007, doi: $10.1088 / 0964-1726 / 21 / 2 / 025007$

[12] Wang, J.; Zhou, S.; Zhang, Z.; Yurchenko, D. (2019). High-performance piezoelectric wind energy harvester with Y-shaped attachments, Energy Conversion and Management, Vol. 181, 645-652, doi:10.1016/j.enconman.2018.12.034

[13] Wang, J.; Tang, L.; Zhao, L.; Zhang, Z. (2019). Efficiency investigation on energy harvesting from airflows in HVAC system based on galloping of isosceles triangle sectioned bluff bodies, Energy, Vol. 172, 1066-1078, doi:10.1016/j.energy.2019.02.002

[14] Dunnmon, J. A.; Stanton, S. C.; Mann, B. P.; Dowell, E. H. (2011). Power extraction from aeroelastic limit cycle oscillations, Journal of Fluids and Structures, Vol. 27, No. 8, 1182-1198, doi:10.1016/j.jfluidstructs.2011.02.003

[15] Singh, K.; Michelin, S.; De Langre, E. (2012). The effect of non-uniform damping on flutter in axial flow and energy-harvesting strategies, Proceedings of the Royal Society A: Mathematical, Physical and Engineering Sciences, Vol. 468, No. 2147, 3620-3635, doi:10.1098/rspa.2012.0145

[16] Huang, D.; Zhou, S.; Litak, G. (2019). Theoretical analysis of multi-stable energy harvesters with high-order stiffness terms, Communications in Nonlinear Science and Numerical Simulation, Vol. 69, 270-286, doi:10.1016/j.cnsns.2018.09.025

[17] Zhou, S.; Cao, J.; Inman, D. J.; Liu, S.; Wang, W.; Lin, J. (2015). Impact-induced high-energy orbits of nonlinear energy harvesters, Applied Physics Letters, Vol. 106, No. 9, Paper 093901, doi: $10.1063 / 1.4913606$

[18] Zhou, S.; Zuo, L. (2018). Nonlinear dynamic analysis of asymmetric tristable energy harvesters for enhanced energy harvesting, Communications in Nonlinear Science and Numerical Simulation, Vol. 61, 271-284, doi:10.1016/j.cnsns.2018.02.017

[19] Zhang, X.; Shan, X.; Shen, Z.; Xie, T.; Miao, J. (2019). A new self-powered sensor using the radial field piezoelectric diaphragm in d33 mode for detecting underwater disturbances, Sensors, Vol. 19, No. 4, Paper 962, 14 pages, doi:10.3390/s19040962 
[20] Shan, X.; Tian, H.; Chen, D.; Xie, T. (2019). A curved panel energy harvester for aeroelastic vibration, Applied Energy, Vol. 249, 58-66, doi:10.1016/j.apenergy.2019.04.153

[21] Liu, Y.; Chen, W.; Liu, J.; Shi, S. (2013). A rectangle-type linear ultrasonic motor using longitudinal vibration transducers with four driving feet, IEEE Transactions on Ultrasonics, Ferroelectrics, and Frequency Control, Vol. 60, No. 4, 777-785, doi:10.1109/TUFFC.2013.2626

[22] Liu, Y.; Chen, W.; Feng, P.; Liu, J. (2012). A square-type rotary ultrasonic motor with four driving feet, Sensors and Actuators A: Physical, Vol. 180, 113-119, doi:10.1016/ j.sna.2012.04.024

[23] Akcabay, D. T.; Young, Y. L. (2012). Hydroelastic response and energy harvesting potential of flexible piezoelectric beams in viscous flow, Physics of Fluids, Vol. 24, No. 5, Paper 054106, doi: $10.1063 / 1.4719704$

[24] Tian, F.-B.; Luo, H.; Zhu, L.; Liao, J. C.; Lu, X.-Y. (2011). An efficient immersed boundarylattice Boltzmann method for the hydrodynamic interaction of elastic filaments, Journal of Computational Physics, Vol. 230, No. 19, 7266-7283, doi:10.1016/j.jcp.2011.05.028

[25] Tian, F.-B.; Luo, H.; Song, J.; Lu, X.-Y. (2013). Force production and asymmetric deformation of a flexible flapping wing in forward flight, Journal of Fluids and Structures, Vol. 36, 149-161, doi:10.1016/j.jfluidstructs.2012.07.006

[26] Wu, Y.; Qiu, J.; Zhou, S.; Ji, H.; Chen, Y.; Li, S. (2018). A piezoelectric spring pendulum oscillator used for multi-directional and ultra-low frequency vibration energy harvesting, Applied Energy, Vol. 231, 600-614, doi:10.1016/j.apenergy.2018.09.082

[27] Tian, F.-B. (2013). Role of mass on the stability of flag/flags in uniform flow, Applied Physics Letters, Vol. 103, No. 3, Paper 034101, doi:10.1063/1.4813006

[28] Tian, F.-B.; Luo, H.; Zhu, L.; Lu, X.-Y. (2010). Interaction between a flexible filament and a downstream rigid body, Physical Review E, Vol. 82, No. 2, Paper 026301, doi:10.1103/ PhysRevE.82.026301

[29] Chen, S.; Doolen, G. D. (1998). Lattice Boltzmann method for fluid flows, Annual Review of Fluid Mechanics, Vol. 30, 329-364, doi:10.1146/annurev.fluid.30.1.329

[30] Song, R.; Shan, X.; Xie, T. (2016). Numerical study of the aerodynamic response and energy harvesting of polyvinylidene fluoride piezoelectric flags in a uniform flow, Journal of the Chinese Chemical Society, Vol. 63, No. 6, 545-552, doi:10.1002/jccs.201500308

[31] Michelin, S.; Doare, O. (2013). Energy harvesting efficiency of piezoelectric flags in axial flows, Journal of Fluid Mechanics, Vol. 714, 489-504 doi:10.1017/jfm.2012.494

[32] Deng, H.-B.; Xu, Y.-Q.; Chen, D.-D.; Dai, H.; Wu, J.; Tian, F.-B. (2013). On numerical modeling of animal swimming and flight, Computational Mechanics, Vol. 52, No. 6, 1221-1242, doi: $10.1007 / \mathrm{s} 00466-013-0875-2$

[33] Zhang, J.; Johnson, P. C.; Popel, A. S. (2007). An immersed boundary lattice Boltzmann approach to simulate deformable liquid capsules and its application to microscopic blood flows, Physical Biology, Vol. 4, No. 4, 285-295, doi:10.1088/1478-3975/4/4/005

[34] Zhu, L.; Peskin, C. S. (2003). Interaction of two flapping filaments in a flowing soap film, Physics of Fluids, Vol. 15, No. 7, Paper 1954, doi:10.1063/1.1582476 